\title{
Tecnologias de gestão dos cuidados nutricionais: recomendações para qualificação do atendimento nas unidades de alimentação e nutrição hospitalares
}

Technology of management of nutritional care: recommendations to qualifying the attendance in hospital food and nutrition services

Anete Araújo de SOUSA²

Rossana Pacheco da Costa PROENÇA ${ }^{3}$

\section{R E S U M O}

\section{Objetivo}

O estudo teve como tema a gestão dos cuidados nutricionais no setor hospitalar, a partir da análise do trabalho do dietista/nutricionista no Brasil e na França, com o suporte da ergonomia e da antropotecnologia.

\section{Métodos}

A pesquisa foi realizada em um hospital francês e um hospital brasileiro. Os dados, analisados qualitativamente, foram obtidos com análise documental, entrevistas semi-estruturadas e observação direta.

\section{Resultados}

Os resultados evidenciaram que as situações de trabalho dos profissionais analisados são influenciadas diretamente pelas características do ambiente externo. No Brasil, estas características ambientais apresentam-se mais desafiantes, em virtude do baixo investimento em saúde, baixos níveis de escolaridade, de poder aquisitivo e acesso aos bens de consumo, baixa formação dos operadores, mudanças no comportamento alimentar e envelhecimento da população. O contexto industrial em evolução, a formação qualificada e a

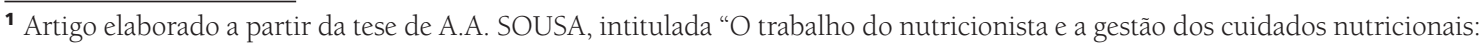
um estudo antropotecnológico em unidades de alimentação e nutrição hospitalares". Programa de Pós-Graduação em Engenharia de Produção, Universidade Federal de Santa Catarina, 2001. 275p.

2 Departamento de Nutrição, Universidade Federal de Santa Catarina. Caixa Postal 476, Campus Universitário, Trindade, 88040-900, Florianópolis, SC, Brasil. Correspondência para/Correspondence to: A.A. SOUSA. E-mail: sousa_anete@hotmail.com; Bolsista CAPES, processo nº BEX 1718/99-1.

3 Departamento de Nutrição e Programa de Pós-Graduação em Engenharia de Produção, Universidade Federal de Santa Catarina. Florianópolis, SC, Brasil.
} 
experiência dos nutricionistas são aspectos positivos para o trabalho destes profissionais. Na França, o alto investimento em saúde e as características organizacionais do setor favorecem os profissionais ao lidarem com o envelhecimento da população, as mudanças no comportamento alimentar, a baixa formação dos operadores, as doenças crônicas não-transmissíveis e os cuidados em domicílio. A formação dos nutricionistas apresenta dificuldades de reconhecimento no setor saúde.

\section{Conclusão}

Neste estudo, elaborou-se um conjunto de recomendações com intuito de contribuir para a gestão dos cuidados nutricionais e para a qualificação e o trabalho do nutricionista no Brasil.

Termos de indexação: ergonomia, antropotecnologia, nutricionista, cuidados nutricionais, unidades de alimentação e nutrição hospitalares.

\section{A B S T R A C T}

\section{Objective}

This study of the nutritional care management in hospital, one in Brazil and the other in France, departs from an analysis of the work of dieticians and nutritionists, supported by ergonomics and anthropotechnology.

\section{Methods}

Data was obtained from documents, semi-structured interviews and direct observation.

\section{Results}

The results suggested that the working conditions of the professionals focused on herein were directly affected by the characteristics of the operational environment. A comparatively more challenging scenario is encountered in Brazil, due to low investments in the health system, lack of education, limited acquisition power and access to consumption, deficient worker background, changes in eating behaviour and population ageing. On the other hand, the developing industrial context, of the country, in association with the nutritionists qualified background and working experience, are positive aspects in their favour. In France, health professionals are assisted by higher investments and better organization of the health system, resulting in improved conditions for the management of population ageing, changes in eating behaviour, low worker background, treatment of chronic non-transmissible diseases, and home health care. The educational background of dieticians is not easily recognized in the health area.

\section{Conclusion}

Recommendations are devised herein in an attempt to contribute to the qualification and as well as work of nutritionists, to the management of nutritional care Brazil.

Index terms: ergonomics, anthropotechnology, nutritionist, nutritional care, hospital food and nutrition services.

\section{N T R O D U ÇÃ O}

O maior desafio para as organizações tem sido a tentativa de acompanhar a evolução de seus ambientes. As organizações ligadas ao setor de saúde, por exemplo, têm enfrentado constan- tes incertezas e riscos, decorrentes da própria necessidade do setor fazer frente aos novos perfis de mortalidade e morbidade da população.

Nas observações feitas por Chaimowicz ${ }^{1}$ com relação à saúde dos idosos brasileiros às vésperas do século XXI, destaca-se que o aumento 
gradual da expectativa de vida das populações, ao longo das últimas décadas, tem trazido à tona o aumento da incidência de uma série de doenças degenerativas, objeto de novas pesquisas e estudos e de redirecionamento no planejamento em saúde.

Além desses aspectos, durante as duas últimas décadas houve um avanço considerável nos conhecimentos relacionados à dietética e à nutrição. Ao mesmo tempo, as fronteiras dessas ciências têm se expandido rapidamente. Um dos exemplos mais atuais refere-se às pesquisas da nutracêutica, ciência que descobriu que os alimentos, por conterem compostos bioativos, auxiliam na prevenção e tratamento das doenças cardiovasculares, câncer e diabetes, dentre outras. Por possuírem um forte poder antioxidante, evitam a oxidação das células, processo este que contribui para o desenvolvimento de doenças crônicas não-transmissíveis².

Ao lado dessas novas descobertas, úteis para a terapia nutricional, o desenvolvimento tecnológico no setor de alimentação coletiva dos hospitais tem caracterizado-se pelas inovações em termos de equipamentos, produtos alimentícios e processos produtivos, além de novos métodos de gerenciamento que se distanciam cada vez mais do fluxo tradicional da maioria das unidades produtoras de refeições ${ }^{3-6}$.

Na França, por exemplo, as discussões sobre a alimentação hospitalar nos encontros da área têm centrado seus esforços na melhoria da prestação dos serviços. O paciente é o centro das preocupações do setor e, dentre outros objetivos, está a revalorização da alimentação, ${ }^{6,7}$.

Simultaneamente, processam-se grandes mudanças na prática dos profissionais ao terem que lidar com esses desafios, os quais exigem novas competências e estratégias de ação a cada dia. Considerando especificamente a prática do nutricionista no setor hospitalar, os contextos apresentados refletem-se diretamente sobre suas atividades no que diz respeito às exigências mentais e, sobretudo, cognitivas.
Alguns estudos sobre a situação de trabalho do nutricionista revelaram um acúmulo de atividades ligadas ao gerenciamento da prestação de serviços, dificultando tanto a sua proximidade com os indivíduos enfermos, como, conseqüentemente, o seu aprofundamento na análise das necessidades terapêuticas e alimentares dos mesmos ${ }^{8,9}$.

Por outro lado, a gestão das atividades relacionadas à produção das refeições é uma ferramenta essencial para a garantia de qualidade do atendimento ao cliente. Tais atividades apresentam, da mesma forma, elementos que distanciam o profissional dos principais objetivos de uma unidade de alimentação e nutrição hospitalar - a prevenção de agravantes e a recuperação do estado nutricional dos pacientes.

Tal distanciamento deve-se, possivelmente, ao modelo de gestão empregada, às exigências de atribuições complexas e à multiplicidade de ações a serem desenvolvidas. Como resultado, esses profissionais, envolvidos no processo de produção das refeições, com toda a complexidade que o cerca (materiais, pessoal, custos, política de produção), vêem-se impossibilitados de uma maior aproximação com o paciente, para a construção de instrumentos que avaliem a efetividade da alimentação no tratamento dos clientes ${ }^{10}$. Além destes aspectos, a interação entre o planejamento da dieta (a prescrição dietética) e a refeição oferecida aos pacientes (a execução da prescrição), apresenta problemas em sua operacionalização.

As considerações acima apontam a necessidade de aprofundar as abordagens dos estudos anteriores, de forma a compreender as condições reais do trabalho do nutricionista e a qualidade das suas intervenções realizadas no setor hospitalar.

Nesta proposta aplicou-se uma abordagem metodológica para avaliar a prática destes profissionais em duas realidades com contextos diferenciados. Na avaliação são enfocados os aspectos relacionados à interação entre a terapia nutricional e a produção de refeições. A 
ergonomia, através da análise ergonômica do trabalho, e a metodologia antropotecnológica foram as ferramentas utilizadas para avaliar as situações de trabalho.

A ergonomia é um campo do conhecimento cujo objetivo é analisar o trabalho, de forma a poder contribuir com a concepção e/ou a transformação das situações e dos sistemas de trabalho ${ }^{11}$. A partir da análise do trabalho real, a ergonomia pode determinar as informações que um operador dispõe para realizar seu trabalho, definindo as características essenciais de uma nova situação de trabalho (os dispositivos técnicos, os meios de trabalho, o ambiente e a organização de trabalho, além das competências e das representações dos operadores).

Outra abordagem utilizada, considerada uma extensão da ergonomia, é a antropotecnologia. Este novo campo de conhecimento nasceu como uma proposta que amplia o entendimento dos sistemas de trabalho e do meio ambiente, em seus diferentes contextos. Tem-se revelado uma ferramenta para a compreensão e alteração dos problemas referentes aos seres humanos, ao trabalho, às novas tecnologias, ao ambiente e aos rumos da sociedade ${ }^{12,13}$.

Considerando a abordagem metodológica e as características do problema, este artigo apresenta os principais aspectos antropotecnológicos determinantes da prática do dietista/ nutricionista no setor hospitalar brasileiro e francês, visando contribuir para a qualificação dos cuidados nutricionais aos clientes/pacientes na realidade brasileira.

\section{CASUÍSTICA E MÉTODOS}

A pesquisa apresentou, como modelo teórico de abordagem, a pesquisa qualitativa ${ }^{14,15}$. Além disso, dada a natureza do estudo, cujo objetivo é analisar de forma detalhada, ambientes e situações particulares, a pesquisa caracterizou-se como estudo de $\operatorname{casos}^{14,16}$. Os estudos de caso compreenderam a prática de dietistas/ nutricionistas e a gestão dos cuidados nutricionais hospitalares, considerando duas realidades distintas - a França e o Brasil.

A pesquisa qualitativa caracteriza-se pela seleção de uma amostra que possibilite "abranger a totalidade do problema investigado em suas múltiplas dimensões". Portanto, o estudo proposto apresentou um critério não numérico para garantir sua representatividade. A seleção dos hospitais e dos atores observados esteve relacionada com a "vinculação mais significativa com o problema a ser investigado"15: os hospitais selecionados caracterizam-se pela gestão dos cuidados nutricionais reconhecidos pela implementação de instrumentos para qualificar o atendimento aos clientes. As etapas da pesquisa foram realizadas inicialmente em um hospital francês, da região parisiense e, posteriormente, em um hospital brasileiro, da região metropolitana de São Paulo; os responsáveis pela gerência geral das unidades de alimentação e nutrição foram selecionados, considerando-se o fornecimento de dados relacionados ao reconhecimento das características do setor e os dados pertinentes ao tema analisado; o dietista/nutricionista selecionado foi o responsável pela gerência da produção de refeições; o dietista/nutricionista do atendimento clínico-nutricional da unidade de internação foi selecionado em função das características do atendimento da clínica (complexidade e diversidade de doenças, tempo de internação e características dos clientes). Destaca-se que a análise, desenvolvida através de estudos de caso, apresenta uma delimitação resultante desta opção metodológica, que permite a replicação, mas não generalização.

Para análise das situações locais e de referência nos dois países, foram utilizadas diferentes técnicas da pesquisa qualitativa, de acordo com as etapas do estudo realizado. Destacam-se a análise documental, as entrevistas semi-estruturadas e a observação direta ${ }^{17}$.

A abordagem antropotecnológica considera que a origem das dificuldades na utilização de uma tecnologia não deve ser 
pesquisada somente nas características individuais dos operadores, mas deve-se considerar tais características em relação aos aspectos sociais, econômicos, geográficos, históricos e antropológicos da situação. Além disso, a comparação entre essas duas situações é um aspecto essencial da metodologia antropotecnológica.

Outra consideração importante da abordagem antropotecnológica é o seu caráter prospectivo. Os meios de análise disponíveis têm como propósito prevenir os efeitos indesejáveis provocados pelas situações futuras na introdução de novas tecnologias.

Os dados, coletados e analisados por Sousa ${ }^{18}$, corresponderam a variáveis referentes ao ambiente externo (contexto social, demográfico e industrial) e, ao ambiente interno (características da organização hospitalar, características da unidade de alimentação e nutrição e características da situação de trabalho).

No presente artigo são priorizados e descritos, os principais aspectos da análise comparativa, culminando com a síntese dos aspectos antropotecnológicos e as recomendações para qualificar os cuidados nutricionais e o trabalho dos nutricionistas para a realidade brasileira.

\section{RESULTADOSE DISCUSS Ã O}

\section{Análise comparativa das situações francesa e brasileira}

Em relação ao Brasil, as características principais do setor de saúde na França correspondem às de um país economicamente desenvolvido graças ao investimento crescente destinado ao setor $(9,50 \%$ do PIB contra 1,71\% para o Brasil), aos gastos hospitalares/habitante (U\$2.010 contra U\$41) e ao número de leitos/mil habitantes (9,2 leitos contra 1,93 leitos). O nível geral de saúde da população francesa situa-se entre os melhores do mundo. Os estabelecimentos hospitalares são majoritariamente públicos, ao contrário da realidade brasileira. Os gastos com alimentação representam 17,00\% dos gastos totais, contra os $23,00 \%$ da população brasileira. Mas, no Brasil, 17,00\% da população encontra-se abaixo da linha de privação de rendimento.

Esses dados são reveladores para compreendermos, tanto a situação dos hospitais públicos nos contextos apresentados pelos dois países, como as condições de saúde e de alimentação das respectivas populações atendidas. Os profissionais de saúde franceses cuidam de uma população com maior poder aquisitivo e acesso aos bens de consumo, com condições satisfatórias de saneamento básico e com alto nível de escolaridade. No Brasil, esses profissionais - incluindo-se neles o nutricionista - defrontam-se com condições inversas. O nutricionista da clínica tem que lidar com um perfil epidemiológico complexo, que demanda cuidados clínico-nutricionais diversificados e estratégias de orientação alimentar e nutricional diferenciadas, devido ao baixo nível de escolaridade da população. O nutricionista da produção de refeições lida com uma diversidade maior de dietas, exigindo maior habilidade para coordenar as diferentes atividades e gerenciar os recursos disponíveis.

Outro aspecto relevante refere-se ao fenômeno crescente de envelhecimento da população, tanto na França como no Brasil. As análises disponíveis consideram que o Brasil enfrentará, nas próximas três décadas, novos problemas nas áreas de saúde e previdência social. No entanto, ao contrário da França, os hospitais brasileiros estão, em geral, pouco capacitados para atender às pessoas idosas.

Outros indicadores que integram este quadro relacionam-se aos padrões de morbi-mortalidade associados à rápida urbanização e industrialização nas situações analisadas. As doenças do aparelho circulatório e os tumores são as maiores causas apontadas. No Brasil acrescenta-se, ainda, a não-superação das doenças infecto-parasitárias. Ao lado desta problemática, a desestruturação do sistema de saúde, com poucos investimentos em atenção básica, traduz-se em um elevado deslocamento 
da população aos centros urbanos, em busca de serviços especializados oferecidos pelo sistema hospitalar. Todos estes aspectos demandam dos profissionais nutricionistas um alargamento das suas competências, não só pelas especificidades relacionadas ao acompanhamento do indivíduo idoso, mas também pela existência de uma demanda diversificada para o setor hospitalar, com diferentes níveis de complexidade em saúde (primário, secundário e terciário).

Outro fenômeno que coloca grandes desafios para os profissionais dos dois países relaciona-se à modificação do comportamento alimentar da população. No ambiente externo dos dois países, diversos aspectos estão relacionados a essas alterações, dentre os quais destaca-se o aumento da taxa de urbanização e, conseqüentemente, uma tendência de simplificação das refeições e de procura de alimentos de fácil preparo.

\section{$\mathrm{O}$ contexto industrial}

O contexto industrial dos dois países coloca aspectos importantes para a atuação dos dietistas/nutricionistas, tendo em vista a melhoria do atendimento aos pacientes; esses aspectos estão, principalmente, relacionados ao suporte tecnológico para o abastecimento de matérias-primas e à diversidade de equipamentos.

O contexto industrial francês tende a favorecer o trabalho desenvolvido pelos dietistas, não só no que concerne à diversidade de matérias-primas, mas, sobretudo, no controle de qualidade em toda a cadeia produtiva, desde a produção de alimentos até o abastecimento. Da mesma forma, os novos conceitos na produção de refeições coletivas, caracterizados pela utilização de produtos pré-elaborados e inovação tecnológica de equipamentos, vêm ganhando ênfase no setor hospitalar francês, principalmente para atender às expectativas do cliente/paciente. Além disso, a informatização dos processos administrativos e a utilização de programas que incluem uma interface de produção de refeições e atendimento clínico-nutricional, revelam um contexto satisfatório para a atuação dos profissionais.

O contexto industrial brasileiro, em especial a região da Grande São Paulo, apresenta aspectos em evolução. A diversidade de produtos na região é considerável. No Brasil, a legislação e a fiscalização sobre os alimentos, de responsabilidade da Secretaria Nacional de Vigilância Sanitária e suas coordenações estaduais, avançaram consideravelmente nos últimos anos. No entanto, $\mathrm{O}$ abastecimento de produtos pré-elaborados apresenta-se, ainda, insatisfatório, assim como o fornecimento de equipamentos, principalmente para o setor público. Muitos dos processos administrativos ainda não são informatizados, o que demanda maior disponibilidade de tempo dos nutricionistas das clínicas e da produção de refeições para o preenchimento de manuais, formulários, mapas e requisições, e para o processamento das informações.

\section{O ambiente interno das unidades analisadas}

As condições organizacionais francesas estão vinculadas a uma política e uma filosofia de atendimento em saúde voltadas para a prevenção. Outro destaque relaciona-se à hierarquização do atendimento, definindo os hospitais em curto, médio e longo período de internação, além do hospital-dia e da hospitalização a domicílio. Na França, a maioria das pessoas com mais de 85 anos beneficia-se deste tipo de hospitalização. Este aspecto, se, por um lado, favorece o trabalho dos dietistas com relação à variabilidade ligada ao paciente (diminuição do número de leitos, diminuição da complexidade e da especificidade das patologias e suas intercorrências), por outro lado, coloca novos desafios para estes profissionais. Estes desafios estão relacionados, sobretudo, às possibilidades do atendimento nutricional em domicílio, associado ao fenômeno de envelhecimento da população.

Para o Brasil, a organização hospitalar reflete as condições apresentadas pelo ambiente 
externo. Na análise da situação de referência, verifica-se uma concentração de recursos tecnológicos de alta complexidade que apresenta, ao mesmo tempo, uma relação com os recursos humanos mais especializados, devendo-se considerar, sobretudo, tratar-se de um hospital universitário. Para os nutricionistas, este tipo de organização hospitalar acaba gerando uma necessidade de maior especialização do conhecimento da nutrição, especialmente no atendimento clínico-nutricional. Com a especialização e com um sistema de saúde que privilegia o curativo, somadas as condicionantes impostas pela situação de trabalho (número de leitos, pressão temporal), possivelmente a procura de técnicas mais especializadas, como a nutrição enteral, passa a ser um dos recursos terapêuticos mais utilizados.

Outro aspecto relevante, diz respeito à formação dos profissionais analisados. Na França, o dietista é, ainda, um profissional formado por escolas técnicas. Os diplomas não são expedidos pelo Ministério da Saúde. Portanto, os dietistas não pertencem à categoria de profissionais de saúde, a exemplo dos médicos, enfermeiros, farmacêuticos, dentre outros, o que implica em uma necessidade de reestruturação para um melhor aproveitamento e reconhecimento do papel destes profissionais junto à área de saúde.

No Brasil, provavelmente, o alto nível de formação dos nutricionistas, abrangendo diferentes áreas de atuação, possibilita a estes profissionais lidarem com as condicionantes ligadas às características do ambiente externo (perfil epidemiológico complexo, demanda diversificada, nível de escolaridade da população e baixo acesso aos bens de consumo, contexto industrial em evolução) e do ambiente interno (estrutura complexa, exigências de certificação, organização temporal das atividades, multiplicidade de ações).

A análise das situações de trabalho evidenciou que, tanto na França, que apresenta condições mais favoráveis ao desenvolvimento do trabalho das dietistas, como no Brasil, as realidades são caracterizadas pela fragmentação das operações, pela imprevisibilidade ligada ao processo de produção de refeições (matérias-primas, pessoas e estrutura), pelas comunicações intensivas com diferentes interlocutores, pelo deslocamento excessivo, pela pressão temporal, pela intensiva exigência mental - sobretudo cognitiva, para perceber as informações provindas de várias fontes (pessoas, formulários, clientes/pacientes), diagnosticar e tomar decisões em curto prazo. No Brasil, estes aspectos são mais evidentes, em virtude das características do ambiente externo.

Tendo em vista as análises comparativas elaboradas, os principais aspectos antropotecnológicos foram sistematizados no Quadro 1.

Considerando as análises realizadas neste estudo, pode-se considerar os seguintes parâmetros na implantação e/ou desenvolvimento de tecnologias de gestão dos cuidados nutricionais aos clientes/pacientes:

- O cliente/paciente deve ser o centro de reflexão das políticas de qualidade alimentar e nutricional nas instituições hospitalares. A alimentação hospitalar como parte dos cuidados hospitalares oferecidos aos clientes, deve integrar qualidades e funções que atendam não só às necessidades nutricionais e higiênicas, como também às necessidades psicossensoriais e simbólicas dos pacientes.

- A sistematização dos diferentes níveis de atendimento nutricional, a partir de uma avaliação do estado nutricional dos clientes/pacientes, deve ser feita no momento da admissão nas unidades hospitalares.

- É necessária a utilização sistemática de instrumentos para avaliação do estado nutricional (evolução dietoterápica) e para avaliação real da ingestão alimentar diária (visitas ao paciente com formulários padrões de ingestão).

- Troca de informações com a equipe de saúde e nutrição: participação de encontros de avaliação do trabalho com a equipe de nutrição (comissão de cardápios, comissão de alimentação e dietética, discussão das enquêtes de satisfação) e com a equipe de saúde (visitas aos pacientes junto com a equipe). 
Quadro 1. Esquema demonstrativo dos principais aspectos antropotecnológicos relevantes das situações de referência na França e no Brasil, para adaptação tecnológica de gestão dos cuidados nutricionais.

\begin{tabular}{cc}
\hline França & Brasil \\
\hline Aspectos antropotecnológicos \\
\hline Investimento no setor saúde \\
\hline $\begin{array}{r}\text { Alto investimento no setor, incluindo recursos humanos } \\
\text { para atendimento humanizado. }\end{array}$ \\
privatização do atendimento por parte dos profissionais de saúde.
\end{tabular}

Aspectos ligados ao comportamento alimentar

- Modificações no comportamento alimentar (simplificação, substituição) associadas à urbanização, exigindo a implementação de programas preventivos em alimentação e nutrição, em todos os níveis de atenção à saúde.

\begin{tabular}{c}
\hline Formação \\
\hline • baixo nível de formação de operadores, exige reformulação \\
\hline $\begin{array}{r}\text { - Alto nível de formação de dietistas nutricionistas, } \\
\text { fortalecimento da formação integrada das áreas de conhecimento. }\end{array}$
\end{tabular}

\begin{tabular}{c} 
Perfil demográfico e epidemiológico \\
\hline • Fenômeno de envelhecimento da população \\
\hline $\begin{array}{cc}\text { - Perfil de morbi-mortalidade associado à urbanização e } \quad \text { Perfil de morbi-mortalidade associados à urbanização e } \\
\text { industrialização (doenças do aparelho circulatório e tumores). }\end{array} \quad$ industrialização, incluindo-se doenças infecto-parasitárias. \\
\hline
\end{tabular}

\section{Contexto industrial (matérias-primas e equipamentos)}

- Contexto industrial satisfatório, legislação fortalecida; evolução de técnicas e conceitos em alimentação coletiva para o atendimento individualizado ao paciente.
- Contexto industrial em evolução, exigindo o fortalecimento da legislação e investimento em equipamentos no setor público.

\begin{tabular}{ll}
\hline Estrutura de saúde \\
\hline $\begin{array}{ll}\text { - Programas de prevenção, crescente hospitalização a domícilio } & \text { - Estrutura de saúde com demanda diversificada. O hospital ainda } \\
\text { e no hospital-dia, sistematização do atendimento (tempo } & \begin{array}{l}\text { é o centro de atenção a todos os níveis de saúde. É necessária a } \\
\text { continuidade da implementação do SUS. }\end{array}\end{array}$ \\
\hline
\end{tabular}

\footnotetext{
- Programas de qualidade centrados no paciente (cuidado e conforto): respeito aos horários da casa, refeitórios, escolha do paciente; estratégias de interação entre produção de refeições e clínicas, incluindo informatização; necessidade de apoio de pessoal administrativo.
}

Sistema de qualidade das UAN

- Programas de qualidade voltados para certificação, exigindo simplificação de rotinas, formação de nível médio, informatização de processos; tendência para produção de refeições com estruturas de controle de qualidade fortalecidas.
- Previsão de folhetos explicativos com recomendações sobre alimentação, nutrição e saúde, além de informações sobre a unidade de alimentação e nutrição, a composição da equipe dos serviços, a estrutura das refeições, etc., para o atendimento aos clientes fora do risco nutricional.

- Adaptação de estruturas para atender às necessidades nutricionais dos clientes em seus diferentes níveis, prevendo-se a organização física, material e humana para a produção normal, dietética, metabólica, enteral, etc.

- Atendimento aos indicadores da qualidade psicossensorial e simbólica, considerandose a percepção dos clientes sobre tais aspectos. Alguns dos indicadores que podem ser avaliados, são: temperatura, utilização de temperos, horário das refeições servidas, o serviço de copa, a apresentação dos pratos, a adaptação das 
refeições às particularidades dos clientes (físicas, culturais), etc.

- Reestruturação dos horários de distribuição das refeições, adequando-os aos horários da casa; projetos com previsão de utensílios mais adaptados aos clientes e a instalação de refeitórios coletivos de acordo com as características individuais dos mesmos.

- Melhoria da qualidade nutricional, organoléptica e higiênica para o fornecimento das refeições envolvendo ações desde a avaliação das matérias-primas até o controle do porcionamento na distribuição das refeições.

- Aperfeiçoamento das unidades de alimentação e nutrição, com estruturas de apoio para os seguintes aspectos: formação de pessoal; desenvolvimento de sistemas informatizados que possam diminuir as atividades administrativas repetitivas; renovação, manutenção preventiva e corretiva das áreas de trabalho, dos equipamentos e materiais.

- Integração de elementos de auditoria de saúde e segurança do trabalho (SST) à auditoria de qualidade interna, já existentes nas estruturas certificadas e nas unidades de alimentação e nutrição que estão em processo de certificação.

Quanto às estratégias de organização e de interação entre o atendimento clínico-nutricional e a produção das refeições, destacam-se:

- Avaliação da qualidade nutricional: conjunto de indicadores, definidos pela unidade e monitorados diariamente, como forma de avaliar o que foi prescrito (prescrição dietética e planejamento de cardápios) e o que foi realizado (produção de refeições).

- Comissão de cardápios mensais: reunião dos nutricionistas ligados à produção de refeições e ao atendimento clínico-nutricional, para a discussão conjunta e planejamento dos componentes dos cardápios.

- Testes de degustação dos alimentos: degustação em pequenas amostras, de todas as preparações, durante e ao final do processamento e antes da expedição aos clientes/pacientes por nutricionistas e operadores.

- Enquetes de opinião ou de satisfação: instrumentos de avaliação dos serviços prestados aos clientes/pacientes, envolvendo nutricionistas do atendimento clínico-nutricional e da produção de refeições.

- Comissão de alimentação e dietética: reunião de avaliação conjunta dos serviços ligados à gestão da logística, na qual se pode avaliar todas as possibilidades de melhorias ligadas ao fluxo operacional entre a produção de refeições e o atendimento clínico-nutricional (horários, equipamentos, materiais e pessoal).

- Ações com os clientes/pacientes: desenvolvimento de dinâmicas de grupo durante as quais se possa explorar o conhecimento dos alimentos, incluindo as formas de preparo e a relação dos alimentos com a enfermidade, considerando a necessidade de implementar ações com caráter preventivo junto aos clientes/ pacientes.

Quanto à organização do trabalho, a gestão dos cuidados nutricionais pode basear-se em estruturas flexíveis, descentralizadas, intensivas em comunicação vertical e, prioritariamente, em comunicação horizontal e lateral (intergerencial).

Nos itens abaixo, encontram-se as bases para uma organização do trabalho para imprimir melhor qualidade aos cuidados nutricionais e ao trabalho do nutricionista no Brasil:

- ênfase no trabalho das equipes organizadas em unidades de trabalho para tomada de decisões conjuntas sobre o atendimento integral aos clientes;

- concepção de gerência como articuladora de vários saberes;

- gestão colegiada com a participação dos gerentes e profissionais das unidades de trabalho.

A partir de uma organização do trabalho com essas características, vislumbram-se os seguintes aspectos: compartilhamento dos resultados do trabalho com a equipe de saúde; 
desenvolvimento de competências técnicas e organizacionais; melhoria da circulação das informações; melhoria dos meios de trabalho (informatização); efeitos positivos para a vida dentro e fora do trabalho e melhor entendimento do cliente/paciente e das suas condições de vida.

\section{Formação dos nutricionistas}

A formação dos nutricionistas revela-se um dos aspectos mais significativos para uma gestão dos cuidados nutricionais centrada no cliente/ paciente. Considera-se a alimentação como um componente cultural importante para indivíduos, mesmo no momento em que se encontram hospitalizados. A refeição, assim como todos os outros procedimentos hospitalares, faz parte da rotina hospitalar de cuidados com os clientes/ pacientes.

Portanto, um acompanhamento nutricional não distanciado dos indivíduos implica mudança de percepção na realidade de atuação. Algumas dessas mudanças seriam de responsabilidade das instituições formadoras, que deveriam buscar a implementação de pressupostos educacionais que incorporassem elementos norteadores na relação pedagógica e que possibilitassem a construção de uma nova concepção de profissional. Seriam estes elementos:

- O reconhecimento da humanidade e sensibilidade presente nos indivíduos: o nutricionista trabalha essencialmente com seres humanos. O alimento por si só perde o sentido se o nutricionista não trabalha a relação do alimento com o ser humano e o ato alimentar. Nessa relação, não somente as dimensões biológica e social são fundamentais; outras relações devem ser percebidas no universo dos indivíduos que estão ao seu redor, sejam clientes/pacientes e familiares, trabalhadores de cozinha, colegas nutricionistas, além da relação simbólica destes indivíduos com o alimento (alimento enquanto prêmio, gula, ansiedade, cultura). Para isso, não basta ser crítico e ser criativo; mais que tudo, é preciso ser sensível para poder intervir, compondo uma parceria com os indivíduos.

- Reconhecimento da existência do conhecimento como uma construção: para compor com os indivíduos, tanto na relação professor-aluno, quanto na relação nutricionistas e outros seres humanos, parece ser fundamental percebermos que os indivíduos trazem consigo a sua história e, com ela, uma bagagem de conhecimentos sobre o mundo, sobre a vida, sobre alimentos, sobre nutrição. Tentar resgatar em cada um este conhecimento, vivenciando novas práticas e criando novos conceitos, é uma tarefa de todos dentro desta relação de troca. O professor, a partir da sua experiência acumulada, pode perceber com maior clareza os caminhos a serem resgatados, compartilhados, mediando, a cada momento, a relação de troca que possa estar existindo entre seus alunos e a realidade proposta para intervenção, ultrapassando, inclusive, a relação teoria-prática-teoria.

- A relação teoria-prática: a construção do nutricionista deveria superar a visão de que teoria deve ser praticada, como se conteúdos teóricos fossem verdades e as práticas fossem um momento em que essas verdades devem ser verificadas, coletadas, classificadas. Para a construção desse profissional, acredita-se que a leitura da realidade pode ser baseada em uma perspectiva, segundo a qual, a relação teoriaprática seja indivisível e a relação entre todos os indivíduos que participem desta construção com o conhecimento, seja de troca.

- A formação do nutricionista não pode perder de vista a necessidade de integração dos conhecimentos básicos sobre alimentos, alimentação, nutrição e seres humanos (aspectos biológicos, socioeconômicos, antropológicos, individuais) com os conhecimentos aplicados nestas áreas. Considera-se que a formação de nutricionistas para uma prática de saúde e nutrição generalista deve continuar sendo a base das novas diretrizes curriculares.

Ressalte-se, todavia, que as dificuldades atuais na prática de nutricionistas para uma 
atuação preventiva parece ser real. Conforme análises da estrutura do sistema de saúde brasileiro, o cliente/paciente não é visto coletivamente. A saúde individual e curativa ainda persiste, inclusive estimulando práticas individuais da equipe de saúde. O nutricionista não apresenta uma retaguarda para a continuidade das suas ações no contexto do bairro (associações de moradores, creches, postos de saúde), da empresa, da escola dos filhos, das cooperativas. No entanto, a integralidade das ações pode ser um dos objetivos a ser buscado na organização das unidades de alimentação e nutrição, através da elaboração de projetos em parceria com as instituições educacionais e de estratégias de organização que englobem os setores de produção de refeições e do atendimento clínico-nutricional e ambulatorial.

\section{Formação contínua dos nutricionistas no trabalho}

Considerando os aspectos sociais, demográficos e industriais do contexto brasileiro, algumas recomendações podem ser pontuadas:

- fortalecimento dos conhecimentos em alimentação e nutrição envolvidos com os cuidados do idoso, não só relacionados aos aspectos nutricionais e higiênico-sanitários, mas, sobretudo, aos aspectos psicossensoriais (organolépticos) e simbólicos da refeição;

- fortalecimento dos conhecimentos em alimentação e nutrição voltados para o grupo de adolescentes, considerando a maior susceptibilidade deste grupo populacional a modificações severas no comportamento alimentar (simplificação, substituição ou anulação de refeições);

- acesso às informações contínuas (banco de dados) sobre os avanços da dietética relacionados à utilização de alimentos para prevenção de doenças degenerativas;

- fortalecimento dos conhecimentos sobre novas tecnologias em alimentação coletiva, considerando o acesso previsível, a médio prazo, de equipamentos que possibilitem aumentar a diversidade das preparações (modos de preparo) e melhorar a sua qualidade nos aspectos nutricionais e organolépticos. Salientam-se, neste ponto, ainda, os conhecimentos atualizados sobre microbiologia de alimentos e controle higiênicosanitário baseados no método Hazard Analysis Critical Control Point (HACCP);

- acesso contínuo às informações sobre a legislação sanitária de alimentos, considerando a introdução de matérias-primas com produção orgânica. Salienta-se ainda, o acesso às informações sobre a utilização de tecnologias de produtos da 4⿳亠口冋 e 5a gerações (pré-elaborados e produtos prontos para consumo), considerando as possibilidades futuras de regulamentação destes produtos.

\section{O N C L US Ã O}

O estudo teve como tema central o trabalho do nutricionista no Brasil e do dietista na França. A abordagem antropotecnológica foi utilizada como recurso para analisar a prática desses profissionais em contextos de implantação de tecnologias de gestão dos cuidados nutricionais. A análise inseriu-se, ainda, numa problemática que considera a necessidade de integralidade das ações desses profissionais através da criação de estratégias de interação e organização, para qualificar os cuidados nutricionais oferecidos aos clientes/pacientes.

Nas recomendações propostas, os aspectos considerados se complementam. Assim, a escolha de uma tecnologia de gestão dos cuidados nutricionais centrada na necessidade humana (dos clientes/pacientes e profissionais) pressupõe, ao mesmo tempo, a criação de atividades de interface e uma organização de trabalho flexível. No mesmo sentido, a formação de nutricionistas pode ser norteada para que se possa aprender e ensinar mutuamente, numa perspectiva de construção e gestão do conhecimento em alimentação e nutrição. 


\section{REFERÊ NCIAS}

1. Chaimowicz F. A saúde dos idosos brasileiros às vésperas do século XXI: problemas, projeções e alternativas. Rev Saude Publica 1997; 31(2): 184-200.

2. Golapan C. Dietetics and nutrition: Impact of scientific advances and development. J Am Diet Assoc 1997; 97(7):737-41.

3. Proença RPC. Aspectos organizacionais e inovação tecnológica em processos de transferência de tecnologia: uma abordagem antropotecnológica no setor de alimentação coletiva [tese]. Florianópolis: Programa de Pós-Graduação em Engenharia de Produção, Universidade Federal de Santa Catarina; 1996.

4. Hôpitaux: une nouvelle génération s'exprime. La Cuisine Collective 1999; 122:12.

5. Quelle cuisine hospitalière por demain? La Cuisine Collective 1999; 125:20-1.

6. Restauration hospitalière: le "repas nutrition" fait son chemin. La Cuisine Collective 1999; 117:10.

7. Ricour C, Rigaud D, Tronchon P. Stratégie soignante: I'alimentation est un soin. Soins 2000; 643.

8. Boog MCF, Rodrigues KRM, Silva SMF. Situação profissional dos nutricionistas egressos da PUCCAMP. Rev Nutr PUCCAMP 1989; 2(1):55-87.

9. Carmo MGT, Holzinger M, Rosa MC, Benvenutti JCLM, Viteritte P. Prática do nutricionista de clínica nos hospitais da Grande Florianópolis-SC. Rev Cienc Saude 1994; 13(1/2):70-81.

10. Sousa AA, Juvêncio JF, Biazus M, Lopes W, Dutra ARA, Proença RPC. O trabalho do nutricionista e a assistência nutricional: um estudo antropotecnológico em uma unidade de internação hospitalar. Anais do $1^{\circ}$ Encontro África-Brasil de Ergonomia; $5^{\circ}$ Congresso Lationoamericano de Ergonomia; $9^{\circ}$ Congresso Brasileiro de Ergonomia e $3^{\circ}$ Seminário de Ergonomia da Bahia; 1999; Salvador. Salvador: Associação Brasileira de Ergonomia; 1999.

11. Rabardel P, Carlin N, Chesnais M, Lang N, Joliff GL, Pascal M. Ergonomie: Concepts et méthodes. Toulouse: Octares Editions; 1998.

12. Santos N, Dutra ARA, Fialho FAP, Proença RPC, Righi CR. Antropotecnologia: a ergonomia dos sistemas de produção. Curitiba: Gênesis; 1997.

13. Wisner A. A inteligência no trabalho: textos selecionados de ergonomia. São Paulo: Fundacentro; 1994.

14. Godoy AS. Introdução à pesquisa qualitativa e suas possibilidades. Rev Admin Empr 1995; 35(2): 57-63.

15. Minayo, MCS. Pesquisa social: teoria, método e criatividade. Petrópolis: Vozes; 1997.

16. Yin RK. Case study research: Design and methods. Beverly Hills: Sage; 1984.

17. Quivy R, Campenhoudt LV. Manual de investigação em ciências sociais. Lisboa: Gradiva; 1992.

18. Sousa AA. O trabalho do nutricionista e a gestão dos cuidados nutricionais: um estudo antropotecnológico em unidades de alimentação e nutrição hospitalares [tese]. Florianópolis: Programa de Pós-graduação em Engenharia de Produção UFSC; 2001.

Recebido para publicação em 6 de maio de 2002 e aceito em 12 de dezembro de 2003 